\section{A year in the life of CMAJ}

\author{
"Accordingly, THE CANADIAN MEDICAL [ASSOCIATION] \\ JOURNAL is issued, as a medium for the expression of all that \\ is best in Canadian Medicine." CMAJ 1911;1:57.
}

$\mathrm{T}$ he past year has been a remarkable one in the life of this scholarly medical journal. The Editor-in-Chief was dismissed, and many of the scientific editors who saw the journal into the year resigned. The report of the CMAJ Governance Review Panel has been delivered to and accepted by the CMA Board. ${ }^{1}$ The new governance structure enshrines editorial independence and has clear lines of communication and accountability. At the time of writing, the search process for a new Editor-in-Chief was nearing completion.

My remit on taking on the editorship of the journal last March was to ensure that $C M A J$ continued to appear every 2 weeks and that its usual high standards were maintained. We have not missed a single issue deadline, weekly submissions are up beyond the number for this time last year and we have included in this issue our annual Holiday Review section, an impossible dream back in July. Readers will judge the enduring quality of the journal for themselves, but during the time of our duress, it was deeply affirming to have authors continue to submit quality work and reviewers carry out timely, thoughtful reviews. Many colleagues delivered encouraging notes and offers of help, some of which were taken up. Even among those few who sent messages critical of the journal or its new editors, we could see the depth of feeling for what CMAJ has come to represent in Canadian medical life.

And now it is time for me to leave my role as interim Editorin-Chief of CMAJ. I owe a deep debt of gratitude to the inhouse editorial staff, who experienced their fair share of the turmoil, to the scientific editors who stayed on and to the new editors who joined us, and to the Interim Editorial Board. I also want to say thank you to the journal's readers, authors and reviewers, without whom the CMAJ would not exist. I am confident that all of these stakeholders will remain committed to full sail for Canada's flagship medical journal as it moves forward under new leadership.

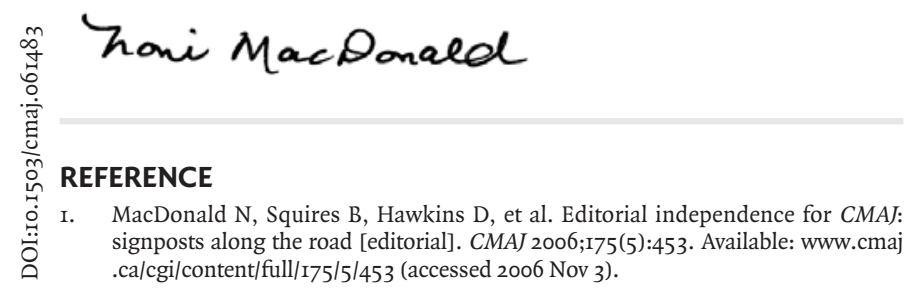

All editorial matter in CMAJ represents the opinions of the authors and not necessarily those of the Canadian Medical Association (CMA) or CMA Media Inc.

\section{Un an dans la vie du JAMC}

«Par conséquent, LE JOURNAL DE L'ASSOCIATION MÉDICALE CANADIENNE est publié comme moyen d'expression de tout ce qu'il y a de mieux en médecine canadienne.» JAMC 1911;1:57.

$\mathrm{L}$ 'année écoulée a été remarquable dans la vie de ce journal médical savant. Le rédacteur en chef a été remercié et un grand nombre des rédacteurs scientifiques qui avaient commencé l'année avec le Journal ont démissionné. Le Groupe d'étude de la gouvernance du JAMC a présenté son rapport au Conseil d'administration de l'AMC, qui l'a accepté1. La nouvelle structure de gouvernance consacre l'indépendance de la rédaction et prévoit une voie claire de communication et de responsabilité. Au moment d'aller sous presse, la recherche d'un nouveau rédacteur en chef tirait à sa fin.

Lorsque j'ai accepté le poste de rédactrice du Journal en mars dernier, ma mission consistait à garantir que le JAMC continuerait de paraître aux deux semaines et que l'on maintiendrait ses normes rigoureuses habituelles. Or, nous n'avons pas raté une seule date de tombée, le nombre de manuscrits reçus chaque semaine dépasse celui que nous avions atteint à la même époque l'an dernier, et nous avons joint à ce numéro notre section annuelle des Fêtes, rêve qui semblait impossible en juillet dernier. Les lecteurs jugeront eux-mêmes de la qualité durable du Journal, mais pendant la période difficile que nous avons vécue, il a été profondément rassurant de voir les auteurs continuer de présenter des travaux de qualité et les examinateurs effectuer leur travail réfléchi en temps opportun. Beaucoup de collègues nous ont fait parvenir des notes d'encouragement et ont offert leur aide, que nous avons parfois acceptée. Même chez les rares auteurs de messages critiques à l'égard du Journal ou de ses nouveaux rédacteurs, nous avons pu ressentir la profondeur de ce que le JAMC en est venu à représenter dans la vie médicale au Canada.

Le moment est maintenant venu de quitter mon rôle de rédactrice en chef intérimaire du JAMC. J'ai une profonde dette de reconnaissance envers le personnel de rédaction interne, dont les membres ont vécu leur part des perturbations, envers les rédacteurs scientifiques qui sont demeurés à bord et envers les nouveaux rédacteurs qui se sont joints à nous, sans oublier le conseil de rédaction intérimaire. Je vous remercie aussi, lecteurs du Journal, auteurs et examinateurs, sans qui le JAMC n'existerait pas. Je suis persuadée que tous ces intervenants demeureront déterminés à voir le principal journal médical du Canada maintenir le cap toutes voiles dehors, un nouveau capitaine à la barre.

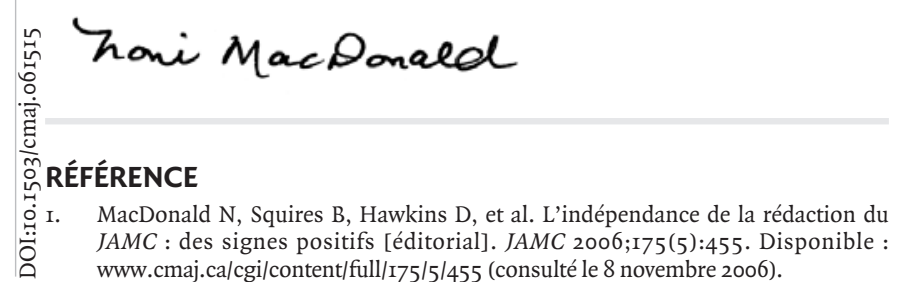

